\title{
Optical binding: Potential energy landscapes and QED
}

\author{
Justo Rodríguez, Luciana C. Dávila Romero and David L. Andrews* \\ Nanostructures and Photomolecular Systems, School of Chemical Sciences, \\ University of East Anglia, Norwich NR4 7TJ, United Kingdom
}

\begin{abstract}
Optical binding can be understood as a laser perturbation of intermolecular forces. Applying state-of-the-art QED theory, it is shown how light can move, twist and create ordered arrays from molecules and nanoparticles. The dependence on laser intensity, geometry and polarization are explored, and intricate potential energy landscapes are exhibited. A detailed exploration of the available degrees of geometric freedom reveals unexpected patterns of local force and torque. Numerous positions of local potential minimum and maximum can be located, and mapped on contour diagrams. Islands of stability and other structures are then identified.
\end{abstract}

Keywords: optical binding, optical matter, quantum electrodynamics, optical manipulation, nanomanipulation, CasimirPolder forces, NEMS

\section{INTRODUCTION}

For studying the mechanical effects of intense light on matter, experiments on nanoscale systems offer considerable advantages over larger systems, although those advantages are to some extent offset by difficulties associated with more significant levels of thermal motion - particularly acute in the case of atomic samples. Such difficulties are commonly overcome by the use of cold atom traps and optical molasses instrumentation, utilizing atomic cooling through momentum exchange with absorbed and emitted photons. In any such context, Maxwell-Bartoli mechanisms represent the operation of optomechanical forces whose origins are well understood, and which characteristically operate on individual particles of matter. For larger particles where conventional optical tweezers can operate, further distinctions in behavior can be drawn on the basis of material composition, by the salient response functions that represent atomic, molecular, dielectric or metallic constitution, for example. In the last of these, the distinctive complex refractive index represents a quality admitting further opportunities to tailor dispersive optical forces, often supplemented by the wellknown exploitation of surface plasmonic effects.

Much recent activity has been prompted by the discovery and verification of optomechanical forces that operate between particles at nanoscale separations. The first proof that intense laser light can produce an optically modified potential energy surface for particle interactions was provided in theoretical work by Thirunamachandran. ${ }^{1}$ That analysis, based on molecular quantum electrodynamics, addressed the issue in terms of a potentially laser-induced perturbation of intermolecular forces. However, the laser intensities that then seemed necessary represented a significant deterrent, and almost a decade passed before Burns et al. ${ }^{2}$ verified the effect experimentally. This latter work also provided the first analysis, for the simplest case of two identical, spherical particles, of the variation of the pair energy with separation - the associated graphs exhibiting striking landscapes of rolling potential energy maxima and minima. Subsequent studies, which showed that optically induced inter-particle forces offer unique opportunities for the controlled optical manipulation of matter, soon generated the hyperbolic terms 'optical binding' and 'optical matter'. Exploiting such interactions, new opportunities for creating optically ordered matter have indeed been demonstrated both theoretically and experimentally. ${ }^{3-10}$ Paraxial wave equations have been adopted to describe optical binding between micron-sized spherical particles, in the presence of counterpropagating beams ${ }^{11}$, 12 , the results being analyzed in terms of the differences between the refractive indices of the spheres and those of the surrounding medium. In an analysis of particles having similar dimensions, Chaumet and Nieto-Vesperinas ${ }^{13}$ also derived results both for isolated spheres, and for spheres near a surface. In the former case they found that inter-particle forces depend significantly on the polarization and wavelength of the incident light, and on particle size. $\mathrm{Ng}$ and $\mathrm{Chan}^{14}$ determined the equilibrium

\footnotetext{
*david.andrews@physics.org
} 
positions in an array of evenly spaced particles, aligned in parallel with the wave-vector of the optical input. Extending the range of applications, more recent studies of optical trapping and binding of cylindrical particles have been carried out by Grzegorczyk et al. ${ }^{15-16}$

\section{QED DESCRIPTION OF OPTICALLY INDUCED PAIR FORCES}

To address the more complex systems that now demand analysis, we start from a recently formulated theory based on quantum electrodynamics (QED). The system state is specified as one in which both the particles and the radiation field are in the ground state; this state couples with other short-lived states in which the electromagnetic field has a non-zero occupation number for one or more radiation modes. The dispersion interaction, traditionally interpreted as a coupling between mutually induced moments, emerges from a fourth-order perturbative calculation based on the exchange of two virtual photons, each created at one particle and annihilated at the other. ${ }^{17-23}$ The theory delivers a result - the CasimirPolder formula - valid for all distances, correctly accounting for the retardation features that lead to a long-range $R^{-7}$ asymptotic dependence on the pair separation $R$. When calculations are performed on a basis state for which the occupation number of at least one photon mode is non-zero, the same, fourth order of perturbation theory gives the leading result; here, the annihilation and creation of one photon from the occupied radiation mode in principle substitute for the paired creation and annihilation events of one of the virtual photons involved in the Casimir-Polder calculation ${ }^{24}$. It is clear that the result of any such calculation on optically conferred pair energies will exhibit a linear dependence on the photon number of the occupied mode. Cast in terms of experimental quantities, this is manifest as an energy shift $\Delta E_{\text {ind }}$ proportional to the irradiance of throughput radiation. Laser-induced coupling forces emerge as the corresponding spatial derivative.

Following the Power-Zienau-Woolley approach, ${ }^{25-28}$ we begin with the Hamiltonian representing the fundamental interaction between the electromagnetic field and a particle $\xi$, in the electric-dipole approximation, as follows;

$$
H_{\mathrm{int}}^{\xi}=-\varepsilon_{o}^{-1} \sum_{\xi} \mu(\xi) \cdot \mathbf{d}^{\perp}\left(\mathbf{R}_{\xi}\right),
$$

where $\boldsymbol{\mu}(\xi)$ and $\mathbf{R}_{\xi}$ denote the electric-dipole moment operator and the position vector of dielectric particle $\xi$. The operator $\mathbf{d}^{\perp}\left(\mathbf{R}_{\xi}\right)$ represents the transverse electric displacement field, expressible in the following general modeexpansion;

$$
\mathbf{d}^{\perp}\left(\mathbf{R}_{\xi}\right)=i \sum_{\mathbf{k}, \lambda}\left(\frac{\hbar c k \varepsilon_{o}}{2 V}\right)^{1 / 2}\left[\mathbf{e}^{(\lambda)}(\mathbf{k}) a^{(\lambda)}(\mathbf{k}) \exp \left(i \mathbf{k} \mathbf{R}_{\xi}\right)-\overline{\mathbf{e}}^{(\lambda)}(\mathbf{k}) a^{\dagger(\lambda)}(\mathbf{k}) \exp \left(-i \mathbf{k} \mathbf{R}_{\xi}\right)\right]
$$

In equation (2.2), $V$ is the quantization volume, and summation is taken over modes indexed by wave-vector $\mathbf{k}$ and polarization $\lambda ; a$ and $a^{\dagger}$ are photon annihilation and creation operators, respectively, and e represents the electric field unit vector, with $\overline{\mathbf{e}}$ being its complex conjugate. Since the laser-induced coupling involves four matter-photon interactions, it requires the application of fourth-order perturbation theory (within the electric dipole approximation), so that the energy shift is given by;

$$
\Delta E_{\text {ind }}=\operatorname{Re}\left[\sum_{t, s, r} \frac{\left\langle i\left|H_{\text {int }}\right| t\right\rangle\left\langle t\left|H_{\text {int }}\right| s\right\rangle\left\langle s\left|H_{\text {int }}\right| r\right\rangle\left\langle r\left|H_{\text {int }}\right| i\right\rangle}{\left(E_{i}-E_{t}\right)\left(E_{i}-E_{s}\right)\left(E_{i}-E_{r}\right)}\right],
$$

$|i\rangle$ being the unperturbed state of the system; $|t\rangle,|s\rangle$, and $|r\rangle$ being virtual states; and $E_{n}$ is the energy of the state $n$. 


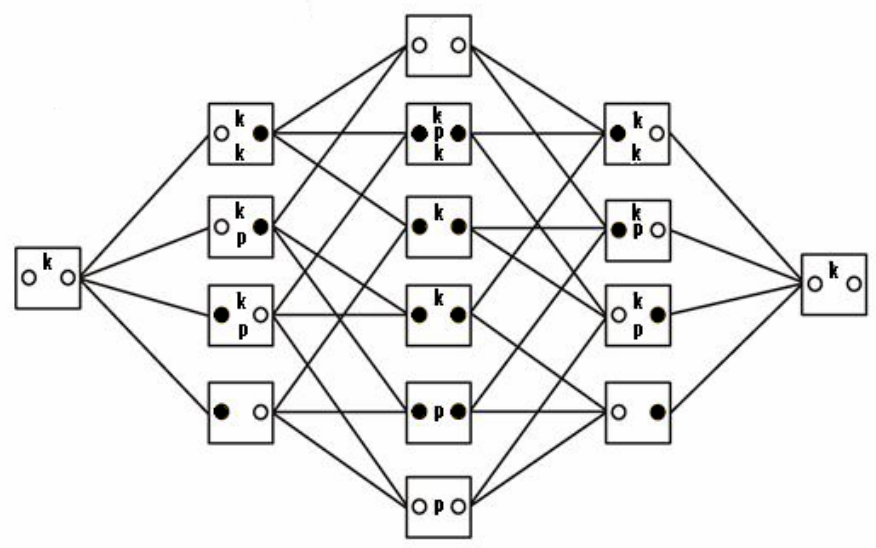

Figure 1: State sequence diagram ${ }^{29}$ for calculation of an interaction energy induced by photon absorption in molecule $A$ (states shown by the circle on the left side of every box), and stimulated emission in another molecule $B$ (right-hand side in each box). The state sequence progresses from left to right across the diagram, each pathway across the figure signifying a distinct contribution to the energy. Open circles denote electronic ground states and closed circles other levels; $\mathbf{k}$ (the laser wave-vector) indicates a laser photon and $\mathbf{p}$ (the virtual photon wave-vector) a virtual photon.

A state sequence diagram of the process, by means of which the laser beam influences the forces between particles, is shown in Fig. 1. This diagram includes all the state sequences that entail the annihilation of laser photons by a particle $A$ and creation by a particle $B$; a similar diagram is also used for the case of creation at $A$ and annihilation at $B$. By calculating the contributions to equation (2.3) from all the pathways exhibited in Fig.1, we obtain the following result for the laser-induced pair energy shift;

$$
\Delta E^{A B}(k, \mathbf{R})=\left(\frac{n \hbar c k}{\varepsilon_{o} V}\right) \operatorname{Re}\left[e_{i}^{(\lambda)} \alpha_{i j}^{A}(k) V_{j k}^{ \pm}(k, \mathbf{R}) \alpha_{k l}^{B}(k) e_{l}^{(\lambda)} \exp (-i \mathbf{k} \cdot \mathbf{R})\right]
$$

Here $n$ is the number of laser photons within a quantization volume $V$, given by the irradiance of the laser beam, $I=n \hbar c^{2} k / V$, and we have introduced the well-known dynamic polarizability tensor $\alpha_{i j}^{\xi}(-k, k)=\alpha_{i j}^{\xi}(k)$; lastly, the fully retarded resonance dipole-dipole interaction tensor features in its general form;

$$
V_{j k}^{ \pm}(k, \mathbf{R})=\frac{\exp (\mp i k R)}{4 \pi \varepsilon_{o} R^{3}}\left\{(1 \pm i k R)\left(\delta_{j k}-3 \hat{R}_{j} \hat{R}_{k}\right)-(k R)^{2}\left(\delta_{j k}-\hat{R}_{j} \hat{R}_{k}\right)\right\}
$$

\section{SPHERICAL PARTICLES}

The analysis is simplest for pairs of spherical particles. Using the angular parameters ( $\phi$ and $\zeta$ ), defined in Fig. 2 the energy shift, equation (2.4), may be written as follows;

$$
\Delta E(\mathbf{k}, \mathbf{R})=\left(\frac{2 I}{\varepsilon_{0} c}\right) \operatorname{Re}\left\{\alpha_{0}^{A} V_{x x}(k, \mathbf{R}) \alpha_{0}^{B}\right\} \cos (k R \sin (\phi) \cos (\zeta)),
$$

From this elegant result, contour plots can be obtained showing the energy surface in a parameter space comprising the two angles and the inter-particle distance $R$. The results give detailed information about the location of the system's 
stability points - see Fig. 3. A host of interesting features emerge even from the examples exhibited here ${ }^{30,31}$. In each energy landscape, local minima distinguish optical binding configurations. The contours intersect the abscissa scale orthogonally, reflecting an even dependence on each angular variable; the variation in the domain $(-\pi / 2, \pi / 2)$ is notionally revealed by unfolding along the distance axis. The physical significance is that a system whose $(k R, \phi, \zeta)$ configuration has $\phi=0$ or $\zeta=0$, but not situated at a local minimum, is always subject to a force drawing it towards a neighboring minimum without change of orientation. For the same reason, there is no torque when $\phi=\pi / 2$ or $\zeta=\pi / 2$. However a system in an arbitrary configuration will generally be subject to both forces and torques, each of which can have either a positive or a negative sign.

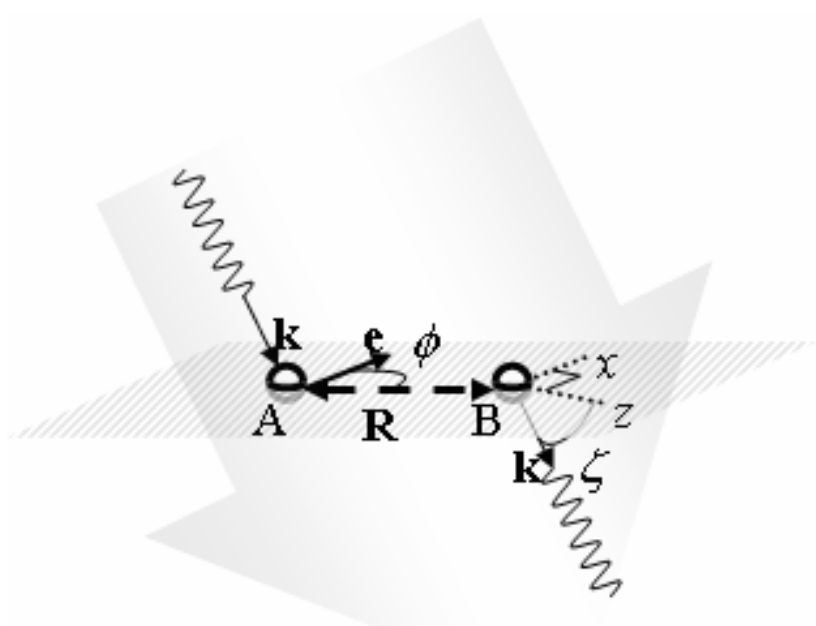

Figure 2: Particles $A$ and $B$, displaced by $\mathbf{R}$, trapped in a polarized laser beam whose throughput is indicated by the broad arrow. The polarization vector, e, defining the $x$-axis, forms an angle $\phi$. with $\mathbf{R}$; together, these vectors define the $x, z$-plane, the wave-vector

$\mathbf{k}$ subtending an angle $\zeta$ on $z$. Wavy lines depict input and output photons, and the dashed line indicates virtual photon coupling

For example, inspection of Fig. 3(a) shows that whilst a pair in the configuration $(4, \pi / 10,0)$ is subject to a torque tending to increase $\phi$. to $\pi / 2$, its trajectory will be accompanied by forces that tend to first increase and then decrease $R$. The details, which will additionally involve changes in $\zeta$, can of course be determined from the total derivative of (2.6). Other features, also exemplified in Fig. 3(a), are off-axis islands of stability such as the one that can be identified (through its darker coloration) at $(10, \pi / 10,0)$. In general the optically induced pair potential provides a prototypical template for the optical assembly of larger numbers of particles, facilitating the optical fabrication of structures of molecules, nanoparticles, microparticles, and colloidal particles.

\section{DISCUSSION}

In the hope of bringing more clarity and precision to the field, the theoretical methods and results we have presented in this paper are based on a robust and thorough quantum electrodynamical analysis of optically induced inter-particle interactions. In this framework it is understood that laser-induced forces and torques between nanoparticles occur by pairwise processes of stimulated photon scattering. The analysis clarifies the fundamental involvement of quantum interactions with the throughput radiation, and also the form of electromagnetic coupling between particles. Relating theory to experiment in this field is perhaps more than usually difficult, but it is a challenge that carries a promise of rich rewards, in the form of new techniques for the nanomanipulation of matter. Part of the problem is that producing suitable conditions for the sought effects generally necessitates the use of specialized cells or optical traps which can generate additional, partly contributory optical effects - each of which can in most cases then compete with optical binding. Another difficulty is that many existing descriptions of optical binding mechanism are a little imprecise, and it is not always clear whether two different descriptions amount to the same, or to potentially competing phenomena. 

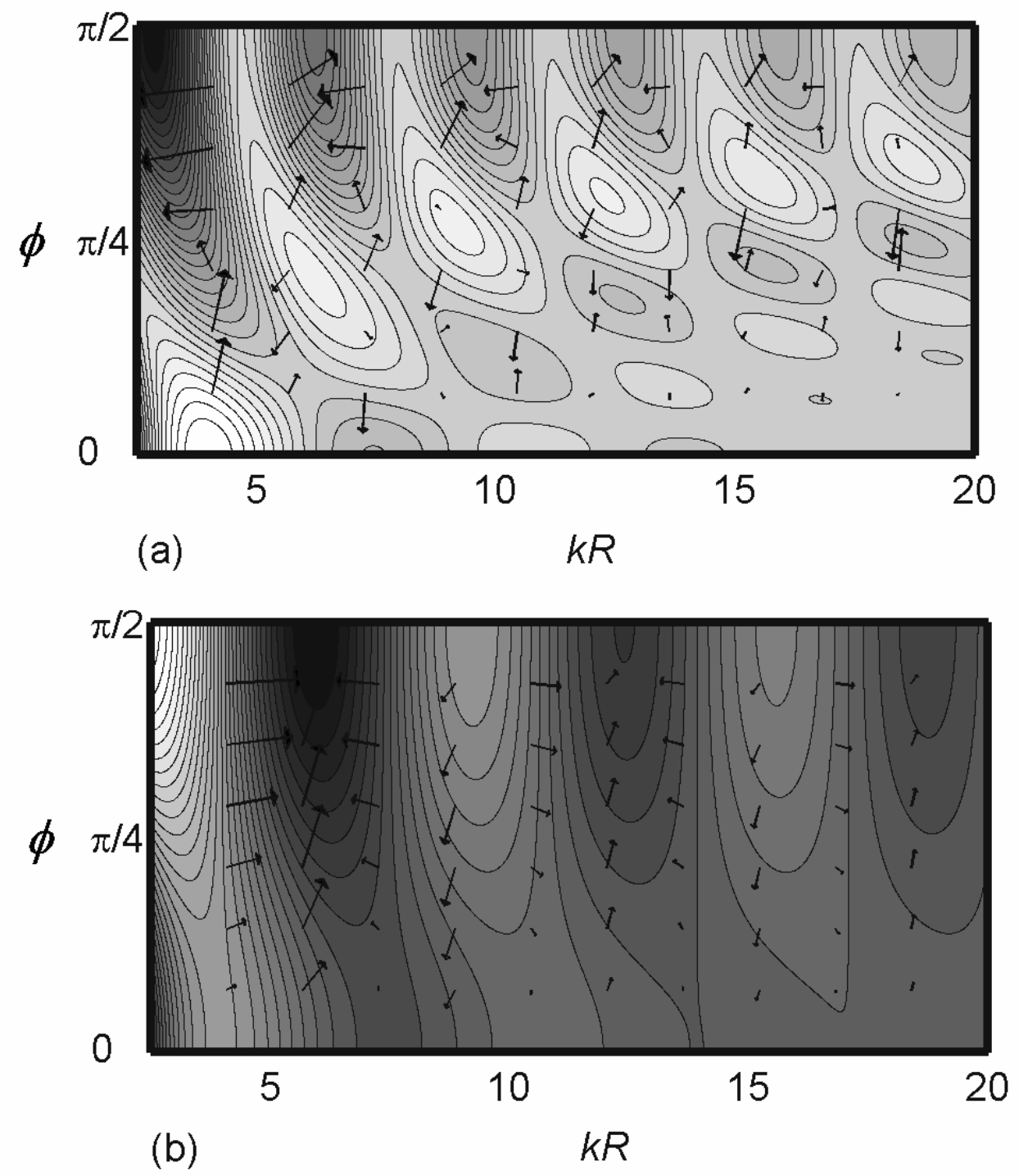

Figure 3: Contour maps of optically induced pair energy. Plots of $\Delta E$ as a function of $\phi$ and $k R$ : (a) $\zeta=0$; (b) $\zeta=\pi / 2$. The variation of $\Delta E$ with $k R$ along the abscissa, $\phi=0$, shows its first two maxima at $k R \sim 4.0,10.5$, the first (non-proximal) minimum, at $k R \sim 7.5$. The abscissa scale typically spans distances $R$ of several hundred nanometers, depending on the value of $k$ (see text). The units of the scale are $\alpha_{o}^{(A)} \alpha_{o}^{(B)} 2 I k^{3} /\left(4 \pi \varepsilon_{o}{ }^{2} c\right)$. On each plot, minima are denoted by the lightest hue and maxima the darkest. Specifically, in (a) the energy scale value for white is 0.11 and black -0.18 ; in (b) white is 0.35 , and black is -0.16 . The arrows point in the direction of greatest descent to a neighboring minimum, their length proportional to the magnitude of the corresponding torques and forces.

In applying the results to nanoparticles whose electronic properties are neither those of one large molecule, nor those of a chromophore aggregate, the molecular properties that appear in the given equations have to be translated into bulk quantities; the polarizability becomes the linear susceptibility, for example. Moreover, account has to be taken of the optical properties of the medium supporting the particles. In most experiments it has been shown that the relative values of the refractive index between the particles and the surrounding medium significantly influence the optical binding phenomena, modifying the positions of stability. With the incorporation of Lorentz field factors, the dependence on $k R$ changes to a dependence on $n(c k) k R$, where the multiplier is the complex refractive index. For example in a liquid 
illuminated by $800 \mathrm{~nm}$ radiation, when the refractive index at that wavelength is 1.40 , the potential energy minimum registered at $k R \sim 7.5$ signifies a pair separation of $670 \mathrm{~nm}$ rather than $960 \mathrm{~nm}$.

The results given here represent the latest output from a program of detailed investigation into the multidimensional potential energy surfaces associated with optical binding. In many of the results - those shown here and others exhibited in recently published reports ${ }^{30,31}$ - these potential energy surfaces exhibit unexpected turning points, producing intricate patterns of local force and torque. Numerous local potential minimum and maximum can be identified, and islands of stability conducive to the formation of rings identified. The major challenge is now to account for the ensemble effects of particles in larger numbers, where there are three distinct aspects to consider. First, there is a need to identify those effects which will very obviously arise as a consequence of the superposition of optically modified pair potentials. Secondly, a full analysis needs to be made of the contributions from multi-particle processes of stimulated scattering, involving the entangled near-field interactions of more than two particles. And finally, since stimulated scattering releases throughput radiation essentially unchanged, multiple processes of stimulated scattering have to be entertained in order to properly address the kind of 1D arrays and 2D optical crystal structures that experiments have so beautifully revealed. We are confident that these challenges for the future will soon bear the fruit of establishing still better and clearer links between theory and experiment.

\section{ACKNOWLEDGEMENT}

The authors are grateful to the EPSRC for financially supporting this work.

\section{REFERENCES}

1. T. Thirunamachandran, "Intermolecular interactions in the presence of an intense radiation-field", Mol. Phys. 40, pp. 393-399, 1980.

2. M. M. Burns, J.-M. Fournier, and J.A. Golovchenko, “Optical binding”, Phys. Rev. Lett 63, pp. 1233-1236, 1989.

3. M. M. Burns, J.-M. Fournier and J. A. Golovchenko, "Optical matter: Crystallization and Binding in intense optical fields", Science, 249, pp. 749, 1990.

4. P. W. Milonni and M. L. Shih, "Source theory of the Casmir force", Phys. Rev. A, 45, pp. 4241, 1992.

5. F. Depasse and J.-M. Vigoureux, "Super-resolution of near field optical microscopy defined form properties of confined electromagnetic waves", J. Phys. D: Appl. Phys. 27, 914, 1994.

6. P. W. Milonni and A. Smith, "van der Waals dispersion forces in electromagnetic fields", Phys. Rev. A. 53, 3484, 1996.

7. P. C. Chaumet and M. Nieto-Vesperinas, "Optical binding of particles with or without the presence of a flat dielectric surface”, Phys. Rev. B 64, 035422, 2001.

8. M. Nieto-Vesperinas, P. C. Chaumet and A. Rahmani, "Near field photonic forces", Phil. Trans. R. Soc. Lond. A 362, 719, 2004.

9. S. K. Mohanty, J. T. Andrews and P. K. Gupta, "Optical binding between dielectric particles", Opt. Express, 12, 2746, 2004.

10. D. McGloin, A. E. Carruthers, K. Dholakia and E. M. Wright, "Optically bound microscopic particles in one dimension", Phys. Rev. E 69, 021403, 2004.

11. N. K. Metzger, E. M. Wright and K. Dholakia, "Visualization of optical binding of microparticles using a femtosecond fiber optical trap", New J. Phys. 8, 139, 2006. 
12. V. Karásek, K. Dholakia and P. Zemánek, "Analysis of optical binding in one dimension”, Appl. Phys. B 84, 149, 2006.

13. P. C. Chaumet and M. Nieto-Vesperinas, "Optical binding of particles with or without the presence of a flat dielectric surface", Phys. Rev. B 64, 035422, 2001.

14. J. Ng and C. T. Chan, "Localized vibrational modes in optically bound structures", Opt. Lett. 31, 2583, 2006.

15. T. M. Grzegorczyk, B. A. Kemp and J.A. Kong, "Trapping and binding of an arbitrary number of cylindrical particles in an in-plane electromagnetic field", J. Opt. Soc. Amer. A, 23, 2324, 2006.

16. T. M. Grzegorczyk, B. A. Kemp and J.A. Kong, "Stable optical trapping based on optical binding forces", Phys. Rev. Lett., 96, 113903, 2006.

17. H. B. G. Casimir and D. Polder, "The Influence of Retardation on the London-van der Waals Forces", Phys. Rev. 73, $360,1948$.

18. P. W. Milonni, “The Quantum Vacuum: An Introduction to Quantum Electrodynamics”, (Academic, San Diego CA, p.54, 1994.

19. D. L. Andrews and L. C. Dávila Romero, "Conceptualization of the Casimir effect”, Eur. J. Phys. 22, 447, 2001.

20. E. A. Power, "Casimir-Polder potential from first principles”, Eur. J. Phys. 22, 453, 2001.

21. G. Jordan Maclay, H. Fearn and P. W. Milonni, "Of some theoretical significance: implications of Casimir effects", Eur. J. Phys. 22, 463, 2001.

22. B. W. Alligood and A. Salam, "On the application of state sequence diagrams to the calculation of the CasimirPolder potential", Mol. Phys. 105, 395, 2007.

23. F. Capasso, J. N. Munday, D. Iannuzzi and H. B. Chan, "Casmir forces and quantum electrodynamics torques; Physics and Nanomechanics”, IEEE J. Select. Topics Quantum Electron. 13, 400, 2007.

24. D. P. Craig and T. Thirunamachandran, Molecular Quantum Electrodynamics (Dover, Mineola, New York, 1998)

25. E. A. Power and S. Zienau, "Coulomb gauge in non-relativistic quantum electrodynamics and the shape of spectral lines", Phil. Trans. R. Soc. A 251, 427, 1959.

26. R. G. Woolley, "Molecular quantum electrodynamics", Proc. R. Soc. A 321, 557, 1971.

27. E. A. Power and T. Thirunamachandran, "On the nature of the Hamiltonian for radiation with atoms and molecules $(e / m c) p \cdot A,-\mu \cdot E$, and all that", Amer. J. Phys. 46, 370, 1978.

28. R. G. Woolley, "Charged particles, gauge invariance, and molecular electrodynamics", Int. J. Quantum Chem. 74, $531,1999$.

29. R. D. Jenkins, D. L. Andrews, and L. C. Dávila Romero, "New diagrammatic methodology for non-relativistic quantum electrodynamics”, J. Phys. B: Atom. Opt. Molec. Phys. 35, 445, 2002.

30. J. Rodríguez, L. C. Dávila Romero and D. L. Andrews, "Optically induced potential energy landscapes", J. Nanophotonics 1, 019503, 2007.

31. L. C. Dávila Romero, J. Rodríguez and D. L. Andrews, "Electrodynamic mechanism and array stability in optical binding", Opt. Commun. (in press, 2007). doi:10.1016/j.optcom.2007.10.026 